\title{
Cell Adhesion Regulates Expression of the Androgen Receptor and Coregulators in Different Prostate Cancer Cells
}

\author{
Jie Zhang ${ }^{1, *}$, Jia-Qing Xiang ${ }^{1}$, Ya-Wei Zhang ${ }^{1}$, Yan-Yan Chen ${ }^{1}$, Xiao-Yan Zhou ${ }^{1}$, Yun Liu ${ }^{2}$ \\ and Sheng $\mathrm{Li}^{2}$ \\ 1 Department of Thoracic Surgery, Shanghai Cancer Hospital, Fudan University, Shanghai 200032, \\ P.R.China; Email: zhangjie2289@hotmail.com, jqxiang2518@yahoo.com.cn, zandy@sh163.net \\ bingya_1020@hotmail.com,zhouxiaoyan@yahoo.com
}

2 Institute for Nutritional Sciences, SIBS, Chinese Academy of Sciences, Shanghai 200031,P.R.China; Email: liuyunbio@hotmail.com, li_sheng69@hotmail.com

*Author to whom correspondence should be addressed; E-mail:zhangjie2289@ hotmail.com, Fax (Tel): + 86-21-6418-3572. Postal address: Department of Surgery, Shanghai Cancer Hospital ,Shanghai 20032, P.R.China

Received: 6 December 2006 / Accepted: 19 February 2007 / Published: 28 February 2007

\begin{abstract}
Prostate cancer cells adhere to a tumor basement membrane, while secretory epithelial cells reside in a suprabasal cell compartment. Since tumor cells are derived from suprabasal epithelial cells, they experience de-novo substratum adhesion in the context of oncogenesis. We therefore analyzed whether cell-matrix adhesion could affect the protein expression and activity of the AR. In this study, AR protein expression declined upon suspension of BPH-1-AR cells, but not in PC-3-AR cells shown by Western blot. In a time course study, BPH-1 cell lost AR expression within 6 hours, and the synthetic androgen, R1881 reduced the loss of AR expression. We further explored the mechanism of AR loss in suspended BPH-1 cells. BPH-1-AR cells underwent apoptosis (anoikis) when suspended for 2 - 5 hours. Suspension did not induce significant apoptosis or decreasing of AR expression in PC-3 cells. Inhibition of apoptosis in suspended BPH-1-AR cells, either by expression of $\mathrm{Bcl}-2$ or Bcl-xl or by treatment with Z-VAD, a caspase inhibitor, prevented loss of AR protein. In contrast, the calpain protease inhibitor, ALLN, accelerated the loss of AR protein expression. Additionally, cell-matrix adhesion changed the expression of coregulators of AR in the mRNA level of prostate cancer cells. Our results demonstrate that AR protein expression was reduced through activation of cell death pathways, and thus indirectly
\end{abstract}


through cell suspension in BPH-AR cells. The activity of AR can also be regulated by adhesion in PC-3-AR and LNCaP cells through affecting the coregulators level.

Key words: androgen receptor; adhesion; suspension; prostate cancer

\section{Introduction}

Cell adhesion to extracellular matrix is critical to maintenance of tissue homeostasis by ensuring that cells remain in their proper tissue environment[1,2]. The normal prostate epithelium consists of a continuous basal cell layer, which is surfaced by secretory cells. Cell adhesion to the substratum and expression of androgen receptor (AR) occur separately in the basal and suprabasal-luminal cell layers, respectively. Thus, in normal epithelium, signaling pathways from cell adhesion and androgen stimulation do not interact[3].

The development of invasive prostate cancer occurs through pre-carcinoma stage, called prostate intra-epithelial neoplasia (PIN). In the early stage of PIN, AR expressing carcinoma cells reside above a continuous basal cell layer. As PIN progresses, the basal cells disappear and carcinoma cells adhere directly to the substratum[4]. Coincidently, the androgen-axis stimulates cancer cell proliferation and survival [5], and AR pathway interacts with cell-matrix adhesion pathway directly in prostate cancer cells. In this paper, we therefore analyzed whether cell-matrix adhesion could affect the expression of the AR and its co-regulators in the prostate cancer cells.

\section{Results and Discussion}

\subsection{Establishment of the cell line model to study cell-matrix adhesion}

LNCaP, an androgen-dependent prostate cancer epithelial cell line, does express endogenous androgen receptor and most of coactivators to assist its activity[6,7], however, it does not adhere to the plate tightly. PC-3, an androgen-independent prostate cancer epithelial cell line, adheres to the plate well, and could be a suitable model for adhesion study after transfected with AR vector. In this study, we mostly used BPH-1 transfected with AR as a representative of pre-invasive prostate cancer to study adhestion to matrix effect. BPH-1 cells are derived from primary human prostate epithelial cultures after immortalization with SV-40 large $\mathrm{T}$ antigen, a potent oncoprotein that abrogates $\mathrm{Rb}$ and p53 function. BPH-1 cells are non-tumorigenic when implanted subcutaneously into nude mice and do not form colonies in soft agar. However, they can become tumorigenic when implanted together with carcinoma-associated fibroblasts. This demonstrates the oncogenic potential of immortalized BPH-1 cells. In our study, we used BPH-1 cell line for two reasons. Our · immunofluorescence staining showed (data not shown) integrin expression of BPH-1 mimic that of prostate cancer tissue as reported by literature[6,8]. Additionally, BPH-1 cells stick to the plate closely through cell-extracellular matrix interaction. After retroviral infections of androgen receptor, BPH-1 and PC-3 cells stably and highly expressed AR showed by western blot and immunofluorescent staining(Figure 1, A,B,time-course study result not shown). 


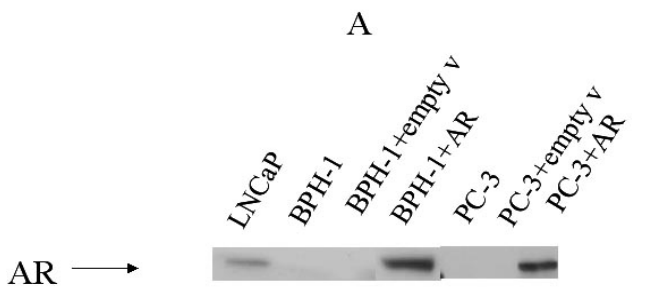

$\mathrm{B}$
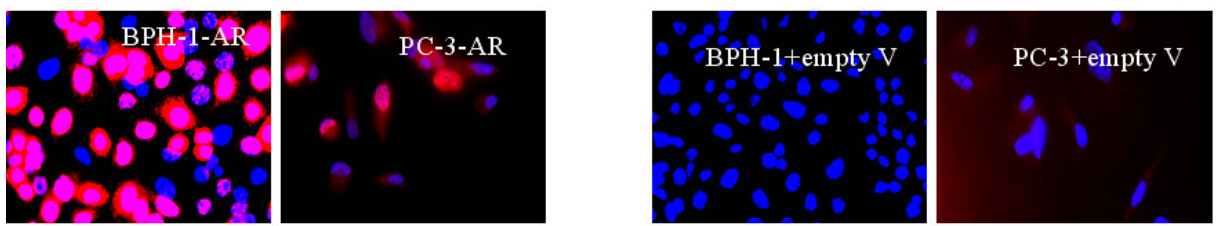

re 1

C

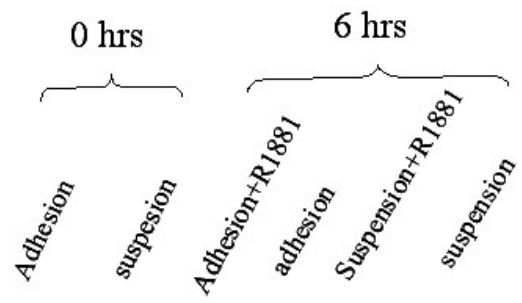

suspension

AR
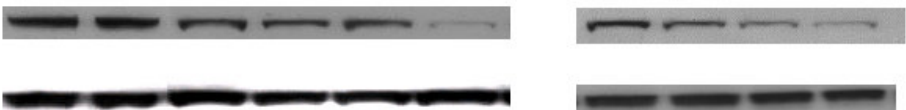

B-actin

$\mathrm{D}$

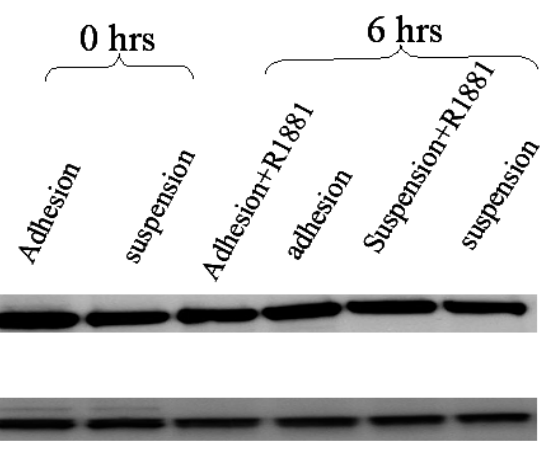

Figure 1. A. BPH-1 and PC-3 cells highly express AR after infection showed by western blot. Western blot of whole cell lysate were probed with anti-AR antibody. Lane 1 is LNCaP cell as positive control, and lane 2-7 are BPH-1, BPH-1 infected with empty vector, BPH-1infected with AR vector, PC -3, PC-3 infected with empty vector, and

PC-3 infected with AR vector, respectively. B BPH-1 and PC-3 highly express AR after infection showed by immunofluorescent analysis. BPH-1 and PC-3 cells were adherent onto coverslips and incubated for overnight. Cell were fixed and stained with anti-AR antibody. More than $80 \%$ of BPH-1 and PC-3 cells expresse AR after infection(left). Empty Pbape vector infection was used for the negative control in this experiment(right). 400X magnification. C Adhesion effect on AR expression in BPH-1-AR cells(left). BPH-1 cells were cultured in charcoal-stripped FCS for at least 48 hours before the experiment. Cells adhering or kept in suspension, treated with R1881 or not were harvested at time point 0 and 6 hours. Western blot of 50 ug cell lysate per lane was probed with mouse anti-AR antibody. The gel was also re-probed with anti-bactin antibody. A time-course study of BPH-1-AR cell in suspension was also conducted(right). D Adhesion effect on AR expression in PC-3-AR cells. Similar with BPH-1-AR cells, adherent or suspended cells either treated with R1881 or not were harvested at time point 0 and 6 hours followed by Western blot for AR expression. 


\subsection{Adhesion effect on AR expression in prostate cancer cells}

BPH-1-AR or PC-3-AR cells were cultured in charcoal-stripped FCS (CS-FCS)for at least 72 hours before the experiment. Cells were adhered on the plate or kept in suspension. AR protein expression declined upon suspension of BPH-1-AR cells, but not in PC-3-AR cells. AR m-RNA level did not change in both BPH-1-AR and PC-3-AR after suspended for 6 hours, so AR was regulated in translational/post-translational level. In a time course study, BPH-1 cell lost AR expression gradually within 6 hours, and the synthetic androgen, R1881 reduced the loss of AR expression(Figure 1,C,D).

\subsection{The mechanism involved in the regulation of AR by adhesion/suspension}

There are many ways in which AR activity can be regulated : modulation of gene expression, protein stability, nuclear translocation, AR transactivation[9,10]. Ligand of the receptor itself , androgen has been known to increase androgen receptor protein stability in various contexts[11,12]. Whether ubiqhitin - proteasome pathway is involved in nuclear receptor regulation is largely studied. Inhibiting proteasomes in human HepG2 and LNCaP cells increased endogenous androgen receptor levels[13]. Lin reported phosphorylation-dependent AR ubiquitylation and degradation by Akt require the involvement of Mdm2 E3 ligase activity[14]. MG132, a $26 \mathrm{~S}$ proteasome inhibitor, suppressed AR transactivation in prostate cancer LNCaP and PC-3 cells[15]. PEST sequence has been identified in AR hinge domain, and it can be degraded by ubiquitin-mediated proteasome system[16]. Signal transduction pathway also plays an important role in regulation of AR[17]. Inhibition of the PI 3kinase/Akt pathway reduced AR protein level correlated with a decrease in AR mRNA in VDEC cells, but not for LNCap cells[18]. Another study found inhibition of PI3K/AKT pathway by LY294002 did not change AR expression, but reduced PSA level [19]. PI3K Akt downstream substrate, FOXO3a can induce AR expression at the protein and mRNA levels[20]. Integrin-linked kinase (ILK) directly interacts with beta integrins and PI3K pathway, so integrin and related factors potentially play a role in AR regulation, There exists interaction between AR and caspase. AR was cleaved by a caspase-3 subfamily protease at Asp146, and this cleavage increased during apoptosis[21]. AR interacted in vivo with an androgen-responsive elements in intron 8 of caspase-2 gene[22]. Knocking down the AR protein level by a small interfering RNA (siRNA) approach resulted in a significant apoptotic cell death[23].

In our present study, we further explored the mechanism of AR loss in suspended BPH-1 cells. BPH-1-AR cells underwent apoptosis (anoikis) when suspended for 2 - 5 hours. Suspension did not induce significant apoptosis or decreasing of AR expression in PC-3 cells(Figure 2 A,B). Inhibition of apoptosis in suspended BPH-1-AR cells, either by expression of Bcl-2 or Bcl-xl or by treatment with Z-VAD, a caspase inhibitor, prevented loss of AR protein(Figure $2 \mathrm{C}$ ). In contrast, the calpain protease inhibitor, ALLN, accelerated the loss of AR protein expression(figure 3). Our results demonstrate that AR protein expression was regulated through activation of cell death pathways, and thus indirectly through cell suspension. It was possible that some caspases released in suspended BPH-1-AR cells, cleaved AR protein without androgen stimulation. 

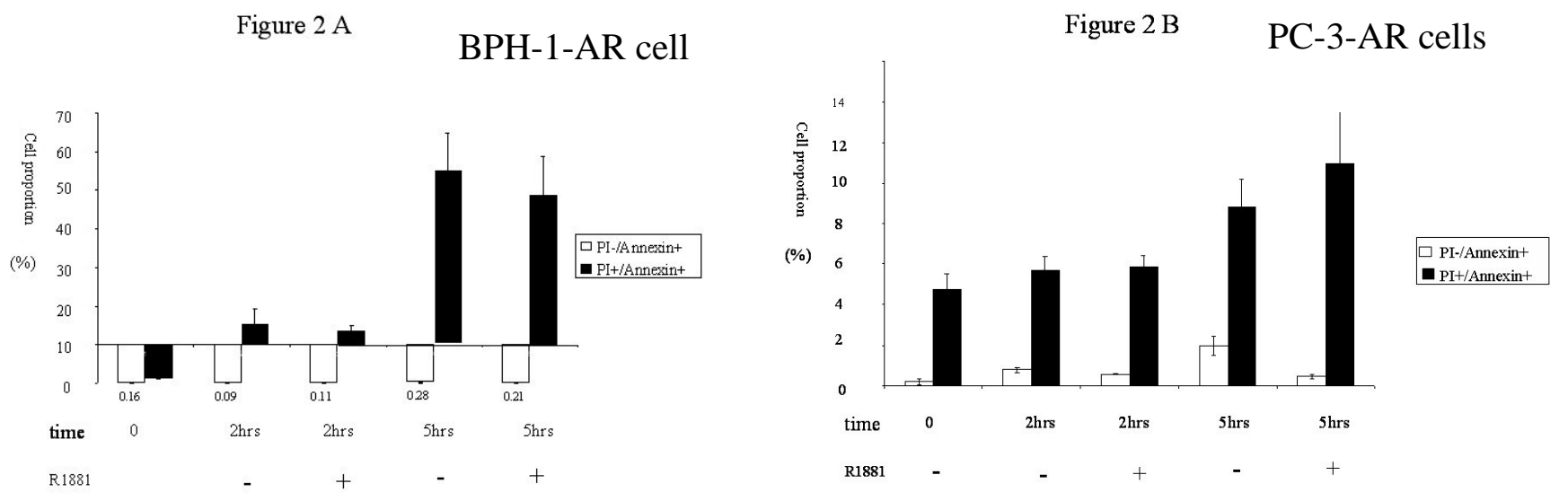

Inhibition of apoptosis pathway can prevent AR from decreasing

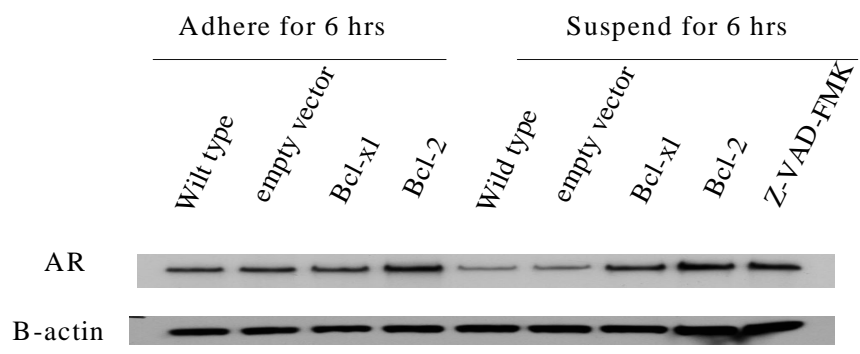

Figure $2 \mathrm{C}$

Figure 2.A. Suspension induced apoptosis in BPH-1-AR cells. BPH-1 cells were suspended for 2 or 5 hours with or without stimulation of R1881 before harvesting. Double staining of Anexin V-FITC and propidium iodide( PI) were done according to manufacturer' instruction(Alexis biochemicals, San Diego, CA).Open bar labeled with Annexin+/PIrepresented early apoptosis, and solid bar labeled with Annexin+/PI+ represented late apoptosis/secondary necrosis.X axis

showed the different time points and $\mathrm{Y}$ axis represented the percentage of early and late apoptotic cells. Triplicate measurements were conducted in this experiment. B. Suspension did not induce significant apoptosis in PC-3-AR. Similar experiment was conducted for PC-3-AR cells, and very few apoptotic cells were identified in suspended PC-3-AR cells. C Inhibition of apoptosis pathway can prevent AR from decreasing. Suspended or adherent BPH-1-AR cells infected with the vectors as indicated were harvested on 6 hours in the absence of R1881 stimulation. Suspended cells was also treated with Z-VAD to block the caspase effect. Levels of AR were examined by Western blot analysis. B-acting was shown as a loading control.

\subsection{The regulation of AR-coregulators in adherent and suspended prostate cancer cell lines.}

AR interacts with basal transcription factors and other proteins, including co-activators and corepressors[24]. When bound to a gene promoter target, such as androgen responsive element (ARE), co-activators, such as SRC-1, SRC-2 and AIB1 accelerates the gene expression and co-repressor inhibits the gene expression. The expression of co-regulators are very important to the activity of AR[25]. 


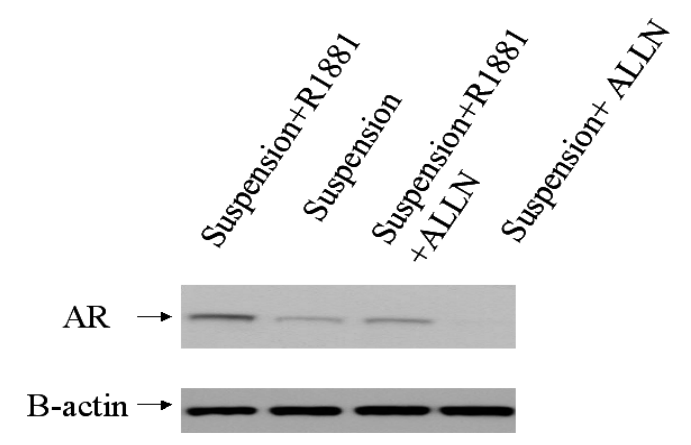

Figure 3 BPH-1 cells were cultured in charcoal-stripped FCS (CS-FCS) for at least 72 hours before the experiment. BPH-1 cells were trypsonized and suspended with indicated treatments for 6 hours. ALLN, a calpain inhibitor, was used at the concentration of $25 \mathrm{mM}$. Western blot of 50 ug cell lysate per lane was probed with anti-AR antibody.

We measured expression of 10 AR co-regulators in adherent and suspended LNCaP and PC-3 prostate cancer cell lines. The following 7 coregulators were measured and found to be significantly higher expressed in suspended PC-3 cells than in adherent PC-3 cells: SRC-1, SRC-2, AIB1, ARA-55, ARA-70, ARA-24 and FHL-2(Figure 4A). LNCaP cells expressed the following co-regulators, SRC-1, SRC-2, ARA-54, ARA-70, ARA-24, NCoA-4, FHL-2 and NCoR-1, and SRC-1 expression was significantly increased in suspended versus adherent cells(Figure 4B). In BPH-1 cells, the expression level of coregulators were very low. From our study, more AR co-regulators were expressed in the suspension than in the adhesion group. This could be one of the preventive mechanisms how suspended cells keep alive.

A Expression of co-regulator in PC-3 -AR cells (relative to b-actin)
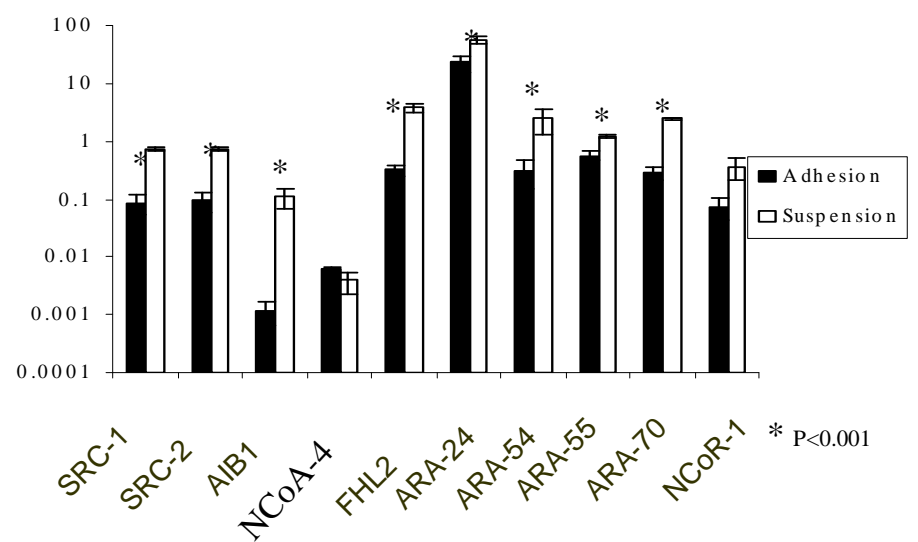


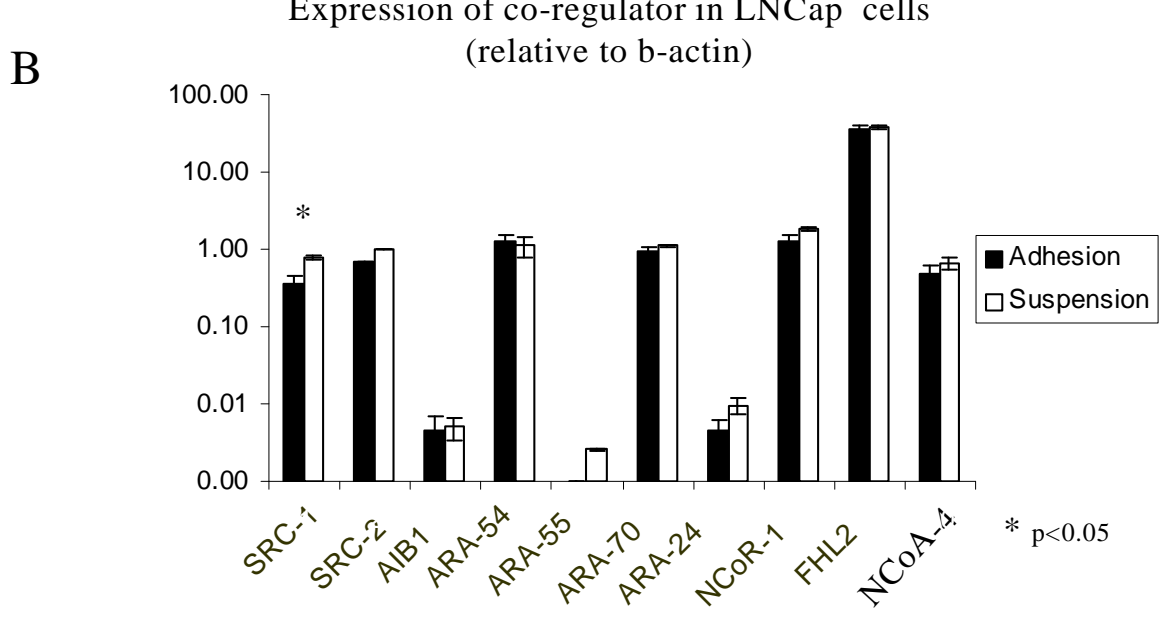

Figure 4. A The regulation of AR-coregulators in adherent and suspended PC-3-AR cells. Real -time PCR was done in triplicate with SYBRA Green Master Mix. ABI 7700 sequence detector and SDS 1.9.1 software were used for PCR reaction and data analysis. The relative values normalized to B-actin were presented. * indicated $\mathrm{P}<0.001$. B.The regulation of AR-coregulators in adherent and suspended LNCap cells. Experiment was similar, and *indicated $\mathrm{P}<0.05$.

\section{Experimental}

\section{Cell cuture}

The BPH-1 cell line was a gift from Simon Hayward, Vanderbilt University and grown in RPMI 1640 / 5\% fetal calf serum. LNCaP, and PC-3 cells were obtained from the ATCC and cultured in RPMI 1640 / 10\% fetal calf serum. Cell culture media and supplements were purchased from GIBCO Invitrogen Corporation (Carlsbad, CA).

\section{Cell lysis and western blot}

Cells were lysed in radioimmuno-precipitation assay (RIPA) buffer. Protein was measured using the Biorad protein determination kit and 50 ug whole cell lysate was analyzed per lane on the gel. Immobilon nitrocellulose membranes were probed with anti-AR antibody (1:200, Santa cruz biotechnology ), followed by goat anti- mouse IgG antibody labeled with $\operatorname{HRP}(1: 10,000$, Santa cruz biotechnology ). B-actin was used as internal control.

\section{Immunofluorescent analysis}

Cells were incubated with anti-AR antibody for one hour at room temperature, followed by incubation with secondary goat anti-mouse IgG coupled to Alexa Fluor-594 (red) (1:500 dilution, Molecular Diagnosis, Eugene, OR) and DAPI (1:2000 dilution ). Fluorescent analysis was conducted with a Nikon fluorescent microscope and images were acquired using a CoolSnap HP camera and Metamorph image analysis software. 
Retroviral infections of androgen receptor, Bcl-2 and Bcl-XL

Phoenix-ampho cells were transfected with Pbabe-puro-AR transiently using Lipofectamine PlusTM reagent( Invitrogen life technologies, Carlsbad, CA). Two days later, retrovirus-containing medium from Phoenix-ampho cells was collected, filtered through 0.45um filter, and added to BPH-1 or PC-3 cells. The infection was repeated in the afternoon to maximize the effect. Two days after the infection, selection using $3 \mathrm{ug} / \mathrm{ml}$ puromycin was conducted for 3 days. The infection of CMMP-Bcl-2 or CMMP-Bcl-XL into BPH-1 cells was conducted similarly as above, except that sorting according to EGFP was conducted after the infection, and high expression of either Bcl-2 or Bcl-XL was confirmed by Western blot.

AR measurement in adherent and suspended cells.

BPH-1-AR or PC-3-AR cells were cultured in charcoal-stripped FCS (CS-FCS) (Life Technologies) for at least 72 hours before the experiment. Cells were adhered on the plate or kept in suspension. After one hour, almost all of the cells adhered to the plate, and $1 \mathrm{n} \mathrm{M}$ of R1881 was added. Adherent and suspended cells either treated with R1881 or not were harvested at time point 0 and 6 hours for Western blot measurement of AR level.

Apoptosis assay by double staining with anexin V-FITC and propidium iodide( PI)

BPH-1-AR and PC-3-AR cells were suspended for 2 or 5 hours before harvesting. Double staining of anexin V-FITC and propidium iodide( PI) were conducted according to manufacturer's instruction ( Alexis biochemicals, San Diego, CA). In brief, $5 \times 10^{5}$ cells were rinsed with $1 \mathrm{x}$ binding buffer provided by kit, stained with $5 \mathrm{ul}$ annexin $\mathrm{V}(20 \mathrm{ug} / \mathrm{ml}$ in Tris $-\mathrm{NaCl})$ and $10 \mathrm{ul} \mathrm{PI} \mathrm{(50ug/ml} \mathrm{in} \mathrm{1x} \mathrm{binding} \mathrm{buffer)}$ in triplicate manner. After incubation at room temperature for 10 minutes, the cells were immediately analyzed in a FACScan (Becton Dickinson, San Jose, USA). 10,000 cells were gated for analysis on the basis of forward and side scatter using FACscan software, and compensation values were adjusted according to annxin V alone and PI staining alone. Annexin+/PI- stands for early apoptosis and annexin+/PI+ stands for late apoptosis/secondary necrosis.

Real-time PCR to measure AR-coregulators in adherent and suspended prostate cancer cell lines

Total RNA was extracted from two independent experiments by Trizol reagent ( Life Technologies, Inc., Rockville, MD ) according to the manufacture's instructions. The first -strand cDNA was synthesized with Superscript II reverse transcriptase and oligo(dT) primers. The primers for coregulators were designed to span different exons to avoid amplification of genomic DNA, and all the primers were tested by routine PCR and 1.5\% agarose gel. 10 primers sets for general and androgen receptor specific coregulators were designed and proved to amplify a single product by routine PCR. Real time PCR was done in triplicate in a final volume of $25 \mathrm{ul}$ containing $0.25 \mathrm{ul}$ forward primer (0.3uM), 0.25ul reverse primer(0.3uM), 12.5 ul SYBRA Green Master Mix (Applied Biosystems , Foster City, CA). ABI 7700 sequence detector( Applied Biosystems, Foster City, CA) and SDS 1.9.1 software were used for PCR reaction and data analysis. Cycle threshold numbers $(\mathrm{Ct})$ of all genes were 
normalized to B-actin. Samples without template were set up to exclude the measurement of primer dimers.

\section{Statistics}

The expression of AR-coregulators in adherent and suspended prostate cancer cell lines were analyzed by independent-samples $\mathrm{T}$ test. $\mathrm{P}<0.05$ was considered to be statistically significant.

\section{Acknowledgements}

We thank Dr Beatrice Knudsen, Dr. Chang Xu and Dr David Hockenbery, from Fred Hutchinson Cancer Research Center, for their supports of experiments and suggestion to the manuscript.

\section{References}

1. Mattila E.; Pellinen T.; Nevo J.; Vuoriluoto K.; Arjonen A.; Ivaska J. Integrins and EGFR coordinately regulate the pro-apoptotic protein Bim to prevent anoikis. Nat. Cell. Biol. 2003, 5, 733-740.

2. Burridge K.; Wennerberg K. Rho and Rac Take Center Stage. Cell 1992, 70, 389-399.

3. Knudsen B.S.; Miranti C.K. The impact of cell adhesion changes on proliferation and survival during prostate cancer development and progression. J. Cell Biochem. 2006, 99, 345-361.

4. Yu H.M.; Frank D.E.; Zhang J.; You X.; Carter W.G.; Knudsen B.S. Basal prostate epithelial cells stimulate the migration of prostate cancer cells. Mol. Carcinog. 2004, 41, 85-97.

5. Heinlein C.A.; Chang C. Androgen receptor in prostate cancer. Endocr. Rev. 2004, 25, 276-308.

6. Cress A.E.; Rabinovitz I.; Zhu W.; Nagle R.B. The alpha 6 beta 1 and alpha 6 beta 4 integrins in human prostate cancer progression. Cancer Metastasis Rev. 1995, 14, 219-228.

7. Rahman M.; Miyamoto H.; Chang C. Expression of Androgen Receptor Coregulators in Prostate Cancer. Clin. Cancer Res. 2004, 10, 2208-2219.

8. Nagle R.B. Role of the Extracellular Matrix in Prostate Carcinogenesis. Journal of Cellular Biochemistry 2004, 91, 36-40.

9. Gelmann E.P. Molecular Biology of the Androgen Receptor. J. Clin. Oncol. 2002, 20, 3001-3015.

10. Yang L.; Xie S.; Jamaluddin M.S.; Altuwaijri S.; Ni J.; Kim E.; Chen Y.T.; Hu Y.C.; Wang L.; Chuang KH.;Wu C.T.; Chang C.Induction of androgen receptor expression by phosphatidylinositol 3-kinase/Akt downstream substrate, FOXO3a, and their roles in apoptosis of LNCaP prostate cancer cells. J. Biol. Chem. 2005, 280, 33558-33565.

11. Krongrad A.; Wilson CM.; Wilson J.D.; Allman D.R.; McPhaul M.J. Androgen increases androgen receptor protein while decreasing receptor mRNA in LNCaP cells. Mol. Cell. Endocrinol. 1991, 76, 79-88.

12. Zhou Z.X.; Lane M.V.; Kemppainen J.A.; French F.S.; Wilson E.M. Specificity of liganddependent androgen receptor stabilization: receptor domain interactions influence ligand dissociation and receptor stability. Mol. Endocrinol. 1995, 9, 208-218.

13. Sheflin L.; Keegan B.; Zhang W.; Spaulding S.W. Inhibiting proteasomes in human HepG2 and LNCaP cells increases endogenous androgen receptor levels. Biochem. Biophys. Res. Commun. 
2000, 276, 144-150.

14. Lin H.K.; Wang L.; Hu Y.C.; Altuwaijri S.; Chang C. Phosphorylation-dependent ubiquitylation and degradation of androgen receptor by Akt require Mdm2 E3 ligase. EMBO J. 2002, 21, 4037 4048 .

15. Lin H.K.; Altuwaijri S.; Lin W.J.; Kan P.Y.; Collins L.L.; Chang C. Proteasome activity is required for androgen receptor transcriptional activity via regulation of androgen receptor nuclear translocation and interaction with coregulators in prostate cancer cells. J. Biol. Chem. 2002, 277, 36570-36576.

16. Brzozowski A.M.; Pike A.C.; Dauter Z.; Hubbard R.E.; Bonn T.; Engelstrom O.; Ohman L.; Greene G.L.; Gustafsson J-A.; Carlquist M. Molecular basis of agonism and antagonism in the oestrogen receptor. Nature 1997, 389, 753-758.

17. Hsu C.L.; Chen Y.L.; Ting H.J.; Lin W.J.; Yang Z.; Zhang Y.; Wang L.; Wu C.T.; Chang H.C.; Yeh S.; Pimplikar S.W.; Chang C. Androgen receptor (AR) NH2- and COOH-terminal interactions result in the differential influences on the AR-mediated transactivation and cell growth. Mol. Endocrinol. 2005, 19, 350-361.

18. Manin M.; Baron S.; Goossens K.; Beaudoin C.; Jean C.; Veyssiere G.; Verhoeven G.; Morel L. Androgen receptor expression is regulated by the phosphoinositide 3-kinase/Akt pathway in normal and tumoral epithelial cells. Biochem. J. 2002, 366, 729-736.

19. Sharma M.; Chuang W.W.;Sun Z.Phosphatidylinositol 3-kinase/Akt stimulates androgen pathway through GSK3beta inhibition and nuclear beta-catenin accumulation. J. Biol. Chem. 2002, 3093530941.

20. Yang L.; Xie S.; Jamaluddin M.S.; Altuwaijri S.; Ni J.; Kim E.; Chen Y.T.; Hu Y.C.; Wang L.; Chuang K.H.; Wu C.T. Chang CInduction of androgen receptor expression by phosphatidylinositol 3-kinase/Akt downstream substrate, FOXO3a, and their roles in apoptosis of LNCaP prostate cancer cells. J Biol Chem 2005, 280, 33558-33565.

21. Ellerby L.M.; Hackam A.S.; Propp S.S.; Ellerby H.M.;Rabizadeh S.; Cashman N.R.; Trifiro M.A.; Pinsky L.; Wellington C.L.; Salvesen G.S.; Hayden M.R.; Bredesen D.E. Kennedy's disease: caspase cleavage of the androgen receptor is a crucial event in cytotoxicity. J Neurochem 1999, 72, 185-195.

22. Rokhlin O.W.; Taghiyev A.F.; Guseva N.V.; Glover RA.;Chumakov P.M.; Kravchenko J.E.; Cohen M.B. Androgen regulates apoptosis induced by TNFR family ligands via multiple signaling pathways in LNCaP. Oncogene 2005, 24, 6773-6784.

23. Liao X; Tang S; Thrasher J.B.;Griebling T.L.; Li B. Small-interfering RNA-induced androgen receptor silencing leads to apoptotic cell death in prostate cancer. Mol Cancer Ther 2005, 4, 505515.

24. Nessler-Menardi C; Jotova I.; Culig Z.; Eder I.E.; Putz T.; Bartsch G.; Klocker H. Expression of Androgen Receptor Coregulatory Proteins in Prostate Cancer and Stromal-Cell Culture Models. Prostate, 2000, 45, 124-131.

25. Kinoshita M.; Nakagawa T.; Shimizu A.; Katsuoka Y. Expression of Androgen Receptor Coregulators in Prostate Cancer. Clin Cancer Res ,2004, 10, 1032-1040.

(C) 2007 by MDPI (http://www.mdpi.org). Reproduction is permitted for noncommercial purposes. 